\section{Avaliando a Saúde Infantil}

\section{em uma Pequena Comunidade:}

O Estudo de Itapirapuã, Vale do Ribeira, SP

Através de inquérito domiciliar, avaliou-se a morbimortalidade e a utilização dos serviços de saúde em crianças menores de cinco anos em um aglomerado rural no Vale do Ribeira - SP. A maioria das crianças pertencia a familias de trabalhadores agricolas com renda inferior a dois salários minimos, vivendo $\mathrm{em}$ precárias condições de habitação e saneamento. $O$ coeficiente de mortalidade infantil foi estimado em 83 por mil nascidos vivos, bastante superior aos dados oficiais para o Município e o Estado. A desnutrição crônica nas formas moderada ou grave atingiu $35 \%$ das crianças. As infecçōes respiratórias e a diarréia foram, repectivamente, os principais motivos de consultas médicas e hospitalizaçóes. $O$ aleitamento materno atingiu a mediana de 14,4 meses, valor jamais descrito no país. As coberturas pré-natal e vacinal foram de apenas $40 \%$ e $65 \%$, respectivamente. $O$ presente estudo evidenciou um enclave com preocupantes niveis de saúde infantil, dentro do estado mais rico do pals. Mostrou, também, que, com um orçamento extremamente limitado e em um curto período de tempo, foi possivel obter dados de grande valia para o diagnóstico comunitário e o planejamento de ações para melhorar a saúde infantil.

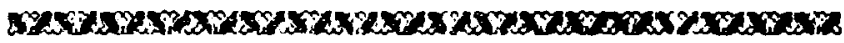

\title{
INTRODUÇÃO
}

A avaliaçāo dos indicadores de morbimortalidade e de utilização dos serviços de saúde em crianças, através de diagnósticos comunitários, tem sido cres-

Cadernos de Saâde Pública, RJ, 6 (4); 455-467, out/dez, 1990

* Bolsista do Conselho Nacional de Desenvolvimento Cientffico e Tecnolbgion (CNPq).

**Professor Adjunto do Departamento de Medicina Social da Faculdade de Medicina da UFPel. 
centemente empregada $(8,15,20,22)$. No Brasil como um todo, inexistem informações confiáveis a respeito da maior parte dos indicadores de saúde materno-infantil $(2,4)$, com exceção de algumas áreas restritas onde foram realizados inquéritos recentes $(15,20$, 22). Estes diagnósticos permitem, se for o caso, sugerir a adoção de medidas relativamente simples e de baixo custo para melhorar a saúde infantil $(8,11)$.

Com o objetivo de transcender parte dessas dificuldades e de diminuir a distância entre os que sabem e os que fazem, o presente estudo foi realizado. Para tanto escolheu-se estudar o Vale do Ribeira, mais particularmente o distrito de Itapirapuā, no município de Ribeira. Esta região, situada no estado de São Paulo, o mais rico do país, é cenário de marcantes desigualdades sociais. Nesse distrito, investigou-se o processo saúde-doença e seus determinantes em crianças menores de cinco anos.

A proposta deste trabalho era demonstrar que, a partir de recursos mínimos e utilizando-se de uma metodologia relativamente simples e rápida, seria possível obter informaçōes de grande valia para o planejamento e implementaçāo de programas em atençäo primária à saúde infantil em pequenas comunidades.

\section{METODOLOGIA}

\section{Descrição da Área Estudada}

$O$ Vale do Ribeira (figura I) está localizado no sul do estado de Sảo Paulo e nordeste do Paraná, englobando 25 municípios. Os 16 municípios paulistas ocupam $2,7 \%$ da superficie do estado e abrigam $0,8 \%$ de sua população $(6,19)$.

Ao redor de 1980 , a maioria da população da regiāo residia na zona rural (18). Para cada dez hectares de terras, quatro se encontravam em situação de posse irregular, o que resultava em conflitos permanentes e graves. Pouco mais de $10 \%$ dessas terras são exploradas economicamente, sendo as principais culturas o milho, feijāo, chá e a banana. Neste mesmo espaço de precariedade e litígios, encontravam-se modernas fazendas de orquídeas destinadas à exportação $(18,19)$.

O município de Ribeira situa-se na região do Alto Vale, a $340 \mathrm{~km}$ da capital paulista, com uma população de 7.639 habitantes (6). Em 1987-1988, o município apresentava importantes deficiências em termos de rede educacional, saneamento básico e energia elétrica. A rede de saúde era constituída de um centro de saúde na sede do município e um Posto de Saúde localizado na zona rural, o qual se destinava a realizar cobertura vacinal e a distribuir suplemento alimentar às gestantes e crianças. Inexistiam clínicas particulares e hospitais (14). 
PESQUISA

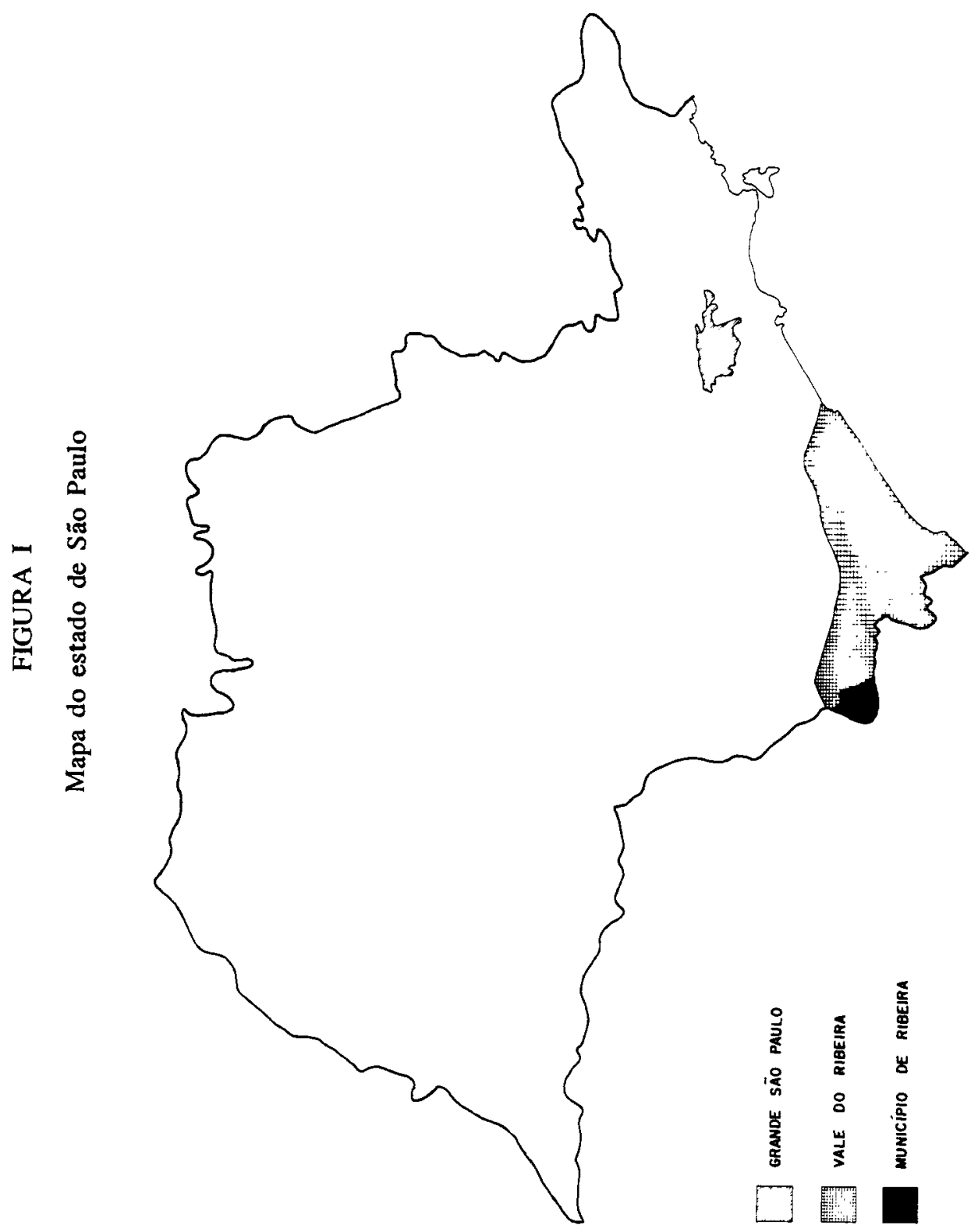


A $29 \mathrm{Km}$ da cidade de Ribeira situa-se o distrito de Itapirapuã, local do presente estudo, que se caracterizava por ser o maior aglomerado rural de todo o município, apresentando serviços, embora que precários, de saneamento básico, telefone, correios, entre outros.

\section{Metodologia do Diagnóstico Comunitário}

No presente estudo, foram visitados todos os 235 domicílios do distrito de Itapirapuã. A coleta de dados foi precedida de uma divisão do distrito em setores geográficos e do mapeamento de todos os domicílios. O trabalho de campo foi realizado em um período de 28 dias, nos meses de julho e agosto de 1988, por um acadêmico do curso de medicina (J.A.C.). Em cada domicílio anotou-se o número total de moradores, o que permitiu o recenseamento da população do distrito. Nos 115 domićlios visitados com crianças menores de cinco anos, aplicaram-se diferentes questionários, os quais haviam sido testados através de estudo-piloto.

$\mathrm{O}$ primeiro deste questionários era aplicado ao chefe da família (pessoa de maior renda) e buscava informaçōes sobre as condições sócio-econômicas e ambientais da família. Um segundo questionário, mais extenso, foi aplicado à mãe ou mulher responsável pela criança, tendo por objetivo obter informaçōes de variáveis maternas (escolaridade, consultas de pré-natal, atendimento ao parto, tipo de parto etc) e variáveis da criança (presença de irmãos menores, hospitalizaçōes de doentes, amamentação e dieta, vacinação, local de nascimento, peso ao nascer, peso e altura atual etc).

Em seguida, a criança era pesada e medida, sendo que aquelas com idade até 24 meses eram medidas deitadas, e as dcmais, na posição ereta.

Foi aplicadc, ainda, um terceiro questionário, sobre a história reprodutiva das mulheres de 15 a 49 anos, com vistas a estimar os coeficientes de mortalidade nos primeiros anos de vida e a expectativa de vida ao nascer.

A codificação era feita pelo entrevistador ao final de cada dia de trabalho. Os questionários foram digitados, utilizando-se o programa $\mathrm{dB}$ ase III e analisados com o pacote SPSS/PC+.

$O$ coeficiente de mortalidade infantil (CMI) foi estimado através da técnica demográfica de Brass-Trussel (9), para cerca de quatro anos antes da coleta de dados (no caso, para 1984). Tabelas de sobrevida foram utilizadas para estimular a prevalência de crianças amamentadas em cada idade (12). 
A ocorrência da diarréia foi definida como um mínimo de três evacuações de fezes líquidas ou amolecidas em um período de 24 horas. Para crianças menores de seis meses, que, freqüentemente, apresentam numerosas evacuações, utilizou-se a definição da mãe.

$\mathrm{O}$ cálculo da cobertura vacinal foi restrito às crianças com 12 meses ou mais. Aceitaram-se como válidas as informações contidas no cartão de vacinação e aquelas fomecidas pela mãe desde que a técnica vacinal fosse descrita adequadamente.

O estado nutricional das crianças foi avaliado através do padrão do National Center for Health Statistics $(N C H S)$, recomendado pela Organização Mundial da Saúde (17). Considerou-se como desnutrida severa a criança que apresentasse três ou mais desvios-padrão (dp) abaixo da mediana, e moderada de 2 a 2,9 desvios-padrâo abaixo da mediana.

\section{RESULTADOS}

Nos 235 domicílios do distrito foram encontradas 203 mulheres com idade entre 15 e 49 anos e 171 crianças menores de cinco anos, estas distribuídas em pouco menos da metade (115) das famílias. Todos os questionários foram respondidos, sendo que apenas uma criança não foi examinada, por encontrar-se hospitalizada por ocasião da coleta de dados.

Pouco mais da metade dessas crianças (56\%) pertenciam ao sexo masculino; $17 \%$ eram primogênitos e em $40 \%$ das 115 famillias havia duas ou mais crianças menores de cinco anos. Nas famílias com menores de cinco anos, o número médio de membros foi de 5,8 , enquanto que, para todo o distrito, a média foi de 5,0 pessoas.

Cerca de $70 \%$ das familias apresentavam renda mensal inferior a dois SMs sendo esta, na grande maioria das vezes (90\%), proveniente de uma única pessoa. $\mathrm{O}$ setor agrícola absorvia $54 \%$ da mão-de-obra ativa, sendo que somente uma em cada dez famílias era proprietária da terra em que trabalhava. Para os demais trabalhadores agrícolas, $47 \%$ eram assalariados temporários (bóias-frias), $40 \%$ eram posseiros e os demais se distribuíam entre proprietários e assalariados permanentes. A remuneração dos trabalhadores agrícolas era marcadamente inferior à dos trabalhadores de outros setores, conforme mostra a tabela I.

Vinte e cinco por cento dos pais não eram alfabetizados e, dos alfabetizados, apenas dois em cada dez apresentavam escolaridade superior ao primário completo.

Para habitação e saneamento, verificou-se que quatro de cada dez moradias eram do tipo paiol ou taipa, não recebiam água tratada e não possuíam qual-

Cadernos de Saúde Pública, RJ, 6 (4): 455-467, out/dez, 1990 
quer tipo de sanitário. Em $85 \%$ dos domicillios dormiam três ou mais pessoas em um mesmo aposento. Essas proporçōes diminuíam à medida em que aumentava o poder aquisitivo das famnlias (figura III). $\mathrm{Na}$ localidade estudada, não existia rede de esgotos.

\section{TABELA I}

Distribuição das famillias conforme o setor de atividade do chefe e a renda mensal, no distrito de Itapirapuã. Ribeira, São Paulo. Julho-Agosto 1988

\begin{tabular}{lrrrrr}
\hline $\begin{array}{l}\text { Setor de atividade do } \\
\text { chefe da famflia }\end{array}$ & \multicolumn{3}{c}{$\begin{array}{c}\text { Renda familiar } \\
\text { mensal em SMs } \%\end{array}$} & $\begin{array}{c}\text { Número de } \\
\text { Crianças }\end{array}$ \\
\hline & $<1$ & 1 a & 1,9 & $\geqslant 2$ & \\
\hline Agrfoola & $43 \%$ & $38 \%$ & $19 \%$ & 74 \\
Serviço Público & $4 \%$ & $64 \%$ & $32 \%$ & 25 \\
Indústria & $7 \%$ & $34 \%$ & $59 \%$ & 18 \\
Prest. Serviços & $13 \%$ & $34 \%$ & $53 \%$ & 15 \\
Outros & $59 \%$ & $43 \%$ & $00 \%$ & 14 \\
\hline Número de crianças & 45 & 58 & 45 & $148(+)$ \\
\hline
\end{tabular}

Obs.: (*) Renda familiar expressa em salários mfnimos mensais

(*) $\mathrm{P}<0,001$

(+) Nómero de crianças com informaçåo sobre renda familar

De cada dez gestantes, somente quatro realizaram cinco ou mais consultas pré-natais, sendo que $20 \%$ não fizeram uma única consulta. Apesar de não ter havido diferença significativa, houve uma tendência a um maior número de consultas à medida que aumentavam renda familiar, escolaridade e idade das mães, de acordo com o demonstrativo em outros estudos $(15,20)$.

Pouco mais da metade das crianças (56\%) nasceram em hospitais e, destes partos, somente $37 \%$ foram atendidos por médicos. Quarenta por cento de todos os partos foram realizados por parteiras, no domicílio. Cesarianas foram realizadas em $21 \%$ de todas as crianças, e em $35 \%$ das nascidas em hospital.

Dos nascidos em hospital, $13 \%$ apresentaram baixo peso ao nascer. No entanto, a maior parte das crianças de baixo nível sócio-econômico estấo excluídas desta análise, por haverem nascido em casa. 
FIGURA II

Condições de habitaçăo e saneamento de acordo com a renda familiar mensal

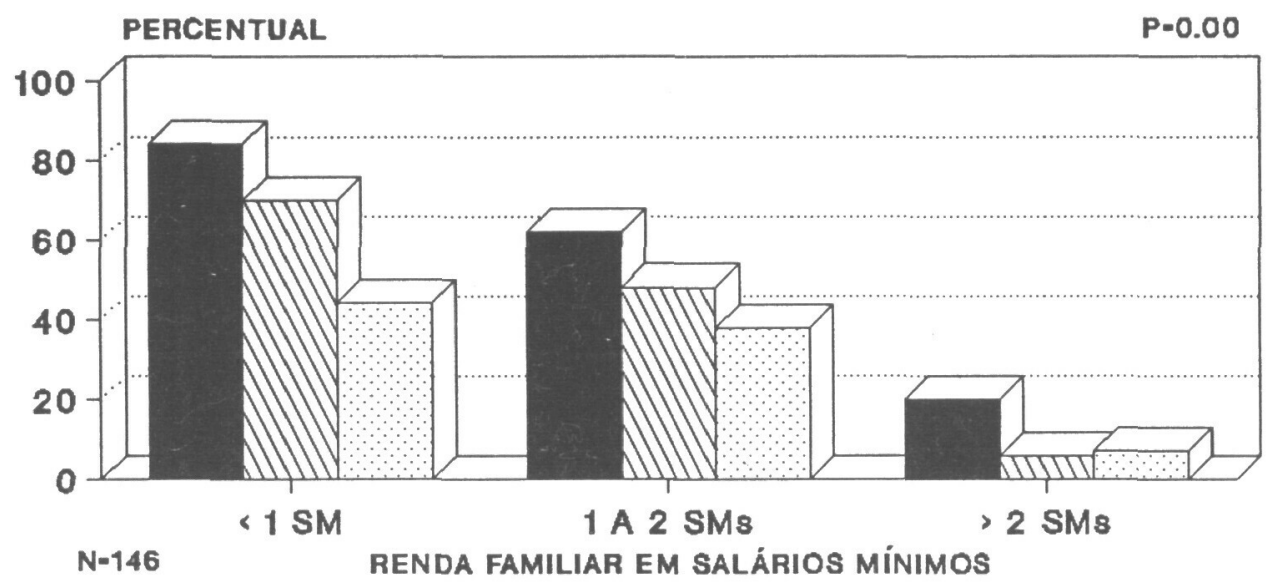

\section{MORADIA PRECÁRIA \\ SEM ÁGUA ENCANADA

No distrito de Itapirapuã, Ribeira-SP. Jul-ago, 1988

Noventa e cinco por cento das crianças foram amamentadas ao seio. Este valor decresceu para $80 \%$ aos seis meses de idade e, ao final do primeiro ano de vida, cerca de $60 \%$ dessas crianças ainda eram amamentadas. A mediana de duração da amamentação foi de 14,4 meses. A figura III mostra esses achados, comparando-os com outras localidades no Brasil.

O CMI estimado foi de $83 / 1000$, bastante superior aos dados oficiais para todo o município de Ribeira $(47 / 1000)$ e para a regiāo (7). A expectativa de vida ao nascer foi de 58,2 anos. Este valor está ligeiramente abaixo da média brasileira $(59,8)$, sendo bastante inferior à estimativa para o estado de São Paulo $(64,5$ anos) (6). Esses dados, no entanto, devem ser analisados com cautela, devido ao tamanho reduzido da amostra.

Cerca de $17 \%$ das crianças haviam sido hospitalizadas nos 12 meses que precederam a entrevista, sendo a diarréia $(6,5 \%)$ e as infecçōes respiratórias $(6 \%)$ os principais motivos. Todas as internaçōes foram realizadas em municípios vizinhos. Pouco mais da metade das crianças $(51 \%)$ havia consultado com médico 
nos últimos três meses, sendo que $47 \%$ das consultas foram por infecçōes respiratórias, seguidas pela diarréia $(16 \%)$.

FIGURA III

Duração da amamentação em Itapirapuã

no estado do Ceará e cidade de São Paulo

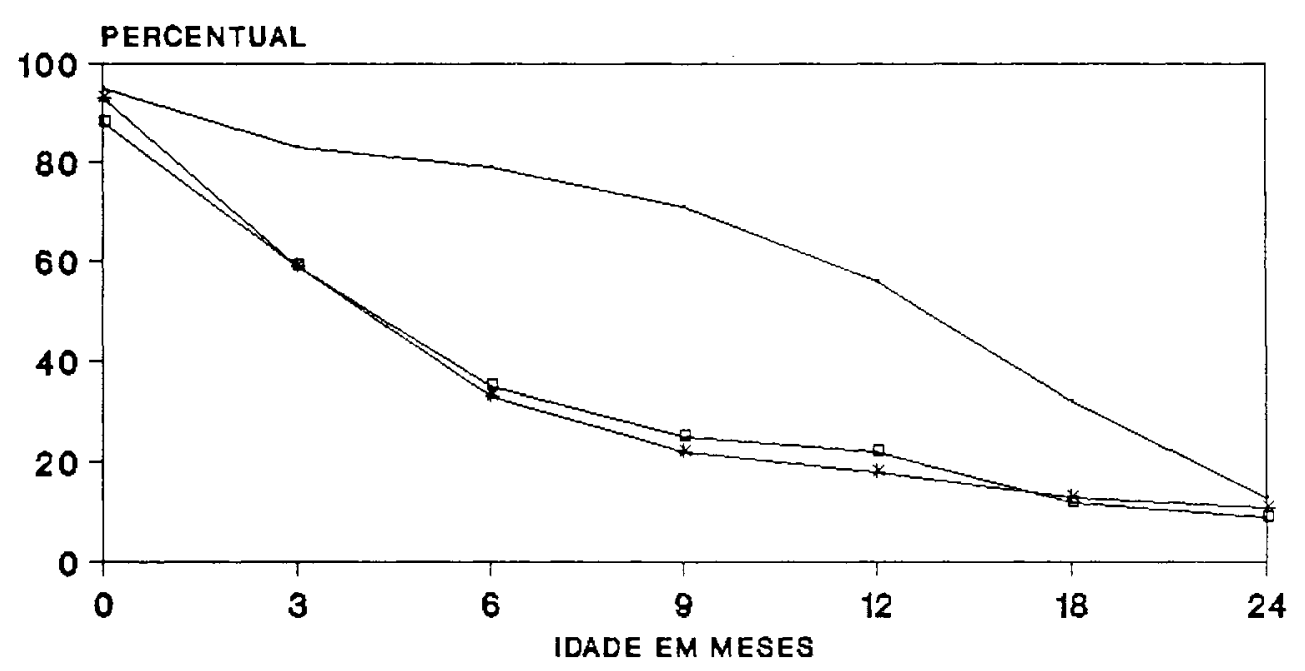

Itapirapuã * São Paulo - Ceará

Quanto a srevalência e incidência de diarréia, verificou-se que $14 \%$ das crianças apresentavam diarréia no dia da entrevista, e $27 \%$ diarréia iniciada na semana anterior. Dessas crianças, apenas três (10\%) receberam terapia de reidrataçăo oral (TRO).

Quanto à prevalência de formas moderadas e severas de desnutrição, $35 \%$ das crianças apresentaram déficit de altura/idade, $9 \%$ de peso/idade e $0,6 \%$ de peso/altura. Estes achados caracterizavam importante presença de desnutrição crônica na população estudada (20).

A cobertura vacinal completa, para maiores de 12 meses, atingiu cerca de $273(65 \%)$ das crianças. Setenta e três e sessenta e sete por cento das crianças receberam, respectivamente, trếs ou mais doses das vacinas sabin e DPT (tétano, coqueluche e difteria). 


\section{DISCUSSĀO}

Para colocar os resultados acima em perspectiva, a tabela II os compara com dados similares da cidade de Săo Paulo (15) e do estado do Ceará (11). Apesar das diferenças de faixa etária, da sazonalidade das doenças e dos métodos empregados nos três estudos, os resultados obtidos para Itapirapuã e o Ceará são bastante similares, revelando as condiçōes precárias em que sobrevivem essas crianças. Os indicadores de morbimortalidade e de utilização de serviços de saúde para o município de São Paulo foram consistentemente melhores.

Entre os resultados da presente pesquisa, quatro merecem especial destaque:

a) o coeficiente de mortalidade infantil de 83 por mil, mais de duas vezes superior ao do município de São Paulo;

b) a prevalência de diarréia de $14 \%$, nas 24 horas que precederam a entrevista, apesar de a pesquisa haver sido realizada durante o inverno; esta prevalência é comparável aos mais altos índices já descritos, não só no Brasil como em outros países (5, $10,11)$;

c) a elevada prevalência de déficit de altura/idade, evidenciando desnutrição crônica;

d) a longa duração do aleitamento, achado pouco freqüente em nosso país (3), sendo substancialmente superior ao observado no município de São Paulo e no Ceará (Figura III); a mediana de 14,4 meses confirma os achados de Rea, em 1975, (18) (mediana de 12,1 meses) na mesma região, e identifica uma das áreas de mais alta duração de amamentação no país.

Os resultados acima evidenciam uma população caracterizada pela má distribuição da terra (16), por péssimas condições de moradia e saneamento e com um serviço de saúde deficiente na prestação de serviços aos mais necessitados. Em última análise, estes fatores podem ser responsabilizados pelas más condições de saúde das crianças.

Algumas medidas de baixo custo e de aplicação relativamente fácil poderiam ser adotadas para reduzir de forma significativa a morbimortalidade dos menores de cinco anos $(8,11,22)$. Estas medidas incluem a promoção da terapia de reidratação oral, o controle das infecçōes respiratórias, aumento da cobertura vacinal (principalmente nas vacinas de doses seriadas), maior assistência à gestação e ao parto, práticas adequadas de desmame, entre outras.

Um aspecto que merece especial atenção é a elevada duraçấo da amamentação, associada a altas prevalências de desnutrição. Estes achados evidenciam a 
necessidade de promover práticas adequadas de desmame com suplementação por alimentos de alta densidade energética (21). Outro ponto a ser melhorado é a atençāo pré e perinatal, onde medidas como o treinamento das parteiras e sua integração no serviço de saúde se fazem necessárias (1).

\section{TABELA II}

Indicadores de distribuição de morbimortalidade infantil e pré-escolar no distrito de Itapirapuã

(Ribeira/SP - 1988), no Ceará (1987)

e na cidade de São Paulo (1984/85)

\begin{tabular}{|c|c|c|c|}
\hline \multirow[b]{2}{*}{ Variáveis } & \multicolumn{3}{|c|}{ Percentuais } \\
\hline & Itapirapuã & Ceará & Såo Paulo \\
\hline Mortalidade infantil $(/ 1000)$ & 83 (a) & 104 (a) & 37 (b) \\
\hline Hospitalizaçōes (12 meses) & 17 & 10 & (c) \\
\hline diartia & 7 & 4 & 3 \\
\hline infec. respiratória & 6 & 3 & 7 \\
\hline \multicolumn{4}{|l|}{ Ocorrencia de diarretia } \\
\hline ultimas 24 horas & 14 & 12 & 2 \\
\hline semana anterior & 27 & 26 & 8 \\
\hline \multicolumn{4}{|l|}{ Desnutriçåo (d) } \\
\hline altura/idade & 35 & 28 & 10 \\
\hline peso/altura & 1 & 1 & 2 \\
\hline peso/idade & 9 & 13 & 6 \\
\hline \multicolumn{4}{|l|}{ Cobertura Vacinal (e) } \\
\hline Sabin & 73 & 73 & 87 \\
\hline Trfplice & 67 & 50 & 85 \\
\hline Sarampo & 84 & 65 & 86 \\
\hline BCG & 94 & 58 & 89 \\
\hline Parto Hospitalar & 56 & 65 & 93 \\
\hline Ocomtacia de cesariana & 35 & 10 & 47 \\
\hline Nåo realizaram pré-natal & 20 & 35 & 7 \\
\hline $\begin{array}{l}\text { Total de crianças } \\
\text { Perfodo }\end{array}$ & $\begin{array}{c}171 \\
\text { JukAgo } \\
1988\end{array}$ & $\begin{array}{c}4513 \\
\text { Out-Dez } \\
1987\end{array}$ & $\begin{array}{c}1016 \\
\text { Abr } 1984 \\
\text { Jun } 1985\end{array}$ \\
\hline
\end{tabular}

(a) Calculo obtido por metodo indireto

(b) Calculo obtido atravks do registro civil

(c) Dado nsomdisponfvel

(d) Formas moderada e severa (DP $>-2$ ), de acordo com o padrăo NCHS.

(e) Crianças com idades entre 12 e 59 meses. 
O presente estudo revelou um enclave de alta morbimortalidade no estado de São Paulo, o mais rico do país. Mostrou, também, que, utilizando-se de uma metodologia relativamente simples e com recursos humanos e financeiros bastante limitados, foi possível obter dados de grande valia tanto para o planejamento e implementação de programas, como, também, para monitorizar o progresso de futuras açôes de saúde.

No entanto, é preciso salientar que estas medidas não substituem a necessidade de transformações profundas na estrutura social de produção, a qual gera desigualdades tão marcadas e submete as crianças de familias trabalhadoras a índices inaceitáveis de morbimortalidade.

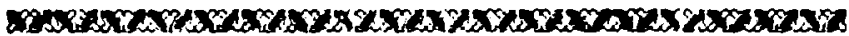

A household survey for evaluating the mortality, morbidity, nutritional status and health services utilization of children aged under five years was carried out in a rural village in Ribeira, São Paulo State. Most children belonged to families of rural laborers earning less than US $\$ 80$ per month and living under inadequate environmental conditions. The infant mortality rate was equal to 83 per thousand and $35 \%$ of the children were affected by moderate or severe stunting, rates which are well above those for the State. The main reasons for clinic attendances and hospital admissions were acute respiratory infections and diarrhea. The median duration of breastfeeding was 14.4 months, the highest reported for Brazil. Only $40 \%$ of the mothers had received antenatal care, and $56 \%$ delivered in a hospital - of whom $35 \%$ had a caesarean section. Vaccine coverage reached $65 \%$ of children aged 12 months or more. This survey revealed an enclave of high morbidity and mortality within Brazil's richest state. It also showed that with a limited budget and within a short time it is possible to obtain valuable information for planning child survival strategies.

\section{AGRADECIMENTOS}

Ao Centro de Pesquisas Epidemiológicas do Departamento de Medicina Social da UFPel; aos professores Amilcar Gigante e Kurt Kloetzel; ao Joaquim e à Jacira por acreditarem nas pequenas idéias. À Elaine Tomasi, pelo carinho e paciência.

Cadernos de Saúde Páblica, RJ, 6 (4): 455-467, out/dez, 1990 
1. ARAUJO J.G., ARAUJO L.J., WALLACE S., and POTTS M. Improving obstetric care in northeast Brazil, (Washington): PAHO BULLETIN, vol 17, no. 3, 1983.

2. ARRUDA J.M., RUTEMBERG N., NORRIS L., FERRAZ E.A. Pesquisa nacional sobre saude materno-infantil e planejamento familiar - Brasil, 1986. Rio de Janeiro: Bemfam/IRD, 1987.

3. BARROS F.C., VICTORA C.G. Breastfeeding and diarrhea in Brazilian Children. Maryland (USA). The Population Council DHS/IRD; number 3, March 1990.

4. BARROS F.C., VICTORA C.G. Avaliando a saúde das crianças: um manual para diagnósticos comunitários de saúde. Brasflia: Unicef, no prelo.

5. BROWN K.H., BLACK R.E., ROMAÑA G.L., and KANASHIRO H.C. Infantfeeding practices and their relationship with diarrhea and other diseases in Huascar (Lima), Peru. Pediatrics 83: 31-40, 1989.

6. Fundação Instituto Brasileiro de Geografia e Estatística 1987/1988. FIBGE. Rio de Janeiro; Gráfica da FIBGE, 1988.

7. Fundação Sistema Estadual de Análise de Dados. Seade. Perfil 1980-1985. Secretaria de Economia e Planejamento do Estado de São Paulo. São Paulo. Gráfica da Seade, 1979.

8. GRANT J.P. Situaçäo Mundial da Infância 1989. Brasłlia: Unicef, 1990.

9. HILL A.G, DAVID P.H. Monitoring Changes in Child mortality: new methods for use in developing countries. London. Health Policy and Planning; 3 (3): 214 - 226. Oxford University Press, 1986.

10. HUTTLY S.R.A., BLUM D., KIRKWOOD B.R., EMEH R.N. and FEACHEM R.G. The epidemiology of acute diarrhea in a rural community in Imo State, Nigeria. London; The Royal Society of Tropical Medicine and Hygiene; 81, 865-870. 1987.

11. Instituto de PI nejamento do Ceará. A saúde das crianças cearenses. Re' itório de um estudo de 8.000 famflias. Unicefl I. plance, $1989,21$.

12. KIRKWOOD B.R. Essentials of medical statistics. London. Blackwel Scientific Publication, Oxford University Press, 1988.

13. Maternity Alliance. One birth in nine. Caesarean section trends since 1978. London: Maternity Alliance, 1983.

14. Levantamento sócio-econômico do munićpio de Ribeira/SP. Ribeira. Associação dos prefeitos da regiāo sudoeste do estado de Sāo Paulo. Prefeitura Municipal de Ribeira, 1989.

15. MONTEIRO C.A. Saúde e nutriçāo das crianças de São Paulo. Um estudo sobre as condiçōes de saúde e nutrição das crianças de São Paulo. São Paulo: Hucitec, 1988.

16. MONTEIRO C.A. Os determinantes da desnutriçāo infantil no vale do Ribeira. Sảo Paulo: Cadernos de pesquisa (Fundaçāo Carlos Chagas), 29, 1979.

Cadernos de Saúde Pública, RJ, 6 (4): 455-467, out/dez, 1990 
17. WATERLOW J.C. et al. The presentation and use of height and weight data for comparing the nutritional status of groups of children under the age of 10 years. Bull $W H O$, $55,1977$.

18. REA M.F. A leitamento materno em núcleos rurais do Vale do Ribeira, Săo Paulo. Dissertação de Mestrado apresentada à faculdade de Medicina da USP. Área de Medicina Preventiva. São Paulo: USP/FM, 1981.

19. Superintendência do Desenvolvimento do Litoral Paulista A questāo fundiária no desenvolvimento do Vale do Ribeira. São Paulo. Secretaria de Estado dos Negócios do Interior. Sudelpa, 1986.

20. VICTORA C.G., BARROS F.C., VAUGHAN J.P. Epidemio logia da Desigualdade. Um estudo longitudinal de 6.000 crianças brasileiras. Sảo Paulo: Hucitec, 1988.

21. VICTORA C.G., VAUGHAN J.P., MARTINES J.C., BARCELOS L.B. Is prolonged breast-feeding associated with malnutrition? Washington; Am J Clin Nutr, 1984.

22. VICTORA C.G., BARROS F.C. et al. Saúde materno-infantil nos estados do Ceará, Rio Grande do Norte e Sergipe: aplicação de uma metodologia para diagnósticos comunitários (submetido para publicação). 